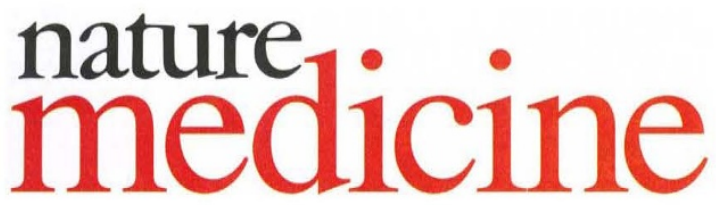

VOLUME 3 - NUMBER 9 • SEPTEMBER 1997

\title{
Guidance on xenotransplantation sought
}

The field of xenotransplantation has reached an important stage in its development. Some clinical trials are underway, and the community is divided as to whether this development is premature. Guidelines aimed at regulating the clinical application of xenotransplantation have been released, but there is concern that these have failed to address all of the issues. There is now the opportunity to listen more closely to all sides of the discussion and to prepare authoritative guidelines that will present a better consensus.

Xenotransplantation is again very much in the news - we have witnessed baboon bone marrow introduced into a patient with advanced AIDS, pig neural cells injected into the brains of patients with Parkinson's disease and pig islet cells given to patients with pancreatic failure.

These are not the first forays into using tissues from animals to help treat sick people; many daring and in hindsight hopelessly cavalier procedures have been performed. However, the new xenografting trials, although by no means endorsed by all interested parties, do not fall into that early category of difficult-todefend experiments. They are carefully considered, cautious attempts to test the low-risk end of the spectrum of xenobased medical interventions and they are set to continue in many countries. Should this concern us? Are safety issues ever likely to be fully satisfied, and is efficacy sufficiently likely that any residual risk is justified? There are no simple answers. Looking at the contributions in this issue of the journal, it is apparent that whereas all manor of procedures involve xenotransplantation, the individual cases are so varied, designed to address such different problems and carry a range of risks, that it is questionable whether any single set of guidelines can adequately accommodate all possibilities.

Michael Thomas and colleagues (page
978) describe the transplantation of a cloned population of bovine adrenocortical cells into immunodeficient mice whose adrenal glands have been removed. As David Sachs points out in an accompanying News \& Views (page 951), although the experiment cannot be considered a direct forerunner to human trials, the successful cloning and expansion of a single cell open the door to in vitro genetic engineering and selection, a more appealing scenario than using a mixed population of cells.

On page 964, Isacson and Breakefield review the opportunities associated with introducing foreign cells into the human brain. The implications for graft rejection are different in this immune privileged site, but so too are the risks associated with using a heterogeneous population of cells. Attention must be paid to the nature of the tissue to be transplanted, where you intend to place it and the source of the tissue. There is a risk of introducing animal-borne retroviruses into the human population, and mice, pigs and primates differ markedly in their endogenous retroviral loads. Fetal tissue is also less likely to have been exposed to the exogenous infectious agents that adult animals inevitably encounter and therefore presents less of a risk to the human recipient.

Fritz Bach and colleagues (page 944) take a step in the direction of higher-risk procedures. Investigating solid organ xenografts (and by no means advocating any clinical application just yet), they consider approaches to avoiding rejection by engineering donor animals to express protective genes and thus avoid as far as possible the need for long-term intensive immunosuppression. Transplantation of vascularized organs from adult mammals probably represents the most extreme risk.

Earlier this year, the US Food and Drug Administration (FDA) produced draft guidelines on xenotransplantation, inviting feedback on their proposals. The response was vocal and included a reaction from an authoritative ad hoc group of concerned parties that cautioned against the use of any xenografting procedures on humans in the absence of much stronger data on the possible risk of cross-species infection, much as the British government's January 1997 report on the subject had concluded. Responsibility for turning the FDA's draft report into finished guidelines has fallen to the National Institutes of Health (NIH), and a final report is expected early in 1998. It is hoped that the steering group will use this time wisely and rather than push through a political agenda that gives the transplant community a green light, they will reconsider the risks. The world's best transplant surgeons are known as innovators, and given the shortage of cadaveric organs, they are eager to push ahead and explore the possibilities of xenografts. It is, however, of tremendous importance that the advice of some of the world's experts on infectious agents is taken into account they know only too well what a virus can do when let loose on the human population. It is also hoped that the steering group will attempt to distinguish the specific risks associated with various procedures and will recognize that introducing a cloned animal cell into a brain tumor is a far cry from transplanting a whole adult organ. It is not clear that this has happened to date.

Few deny the opportunity that xenotransplantation provides. However, there are clear paths forward that balance the opportunity with the risks. Guidelines are needed to steer the more daring practitioners into quieter waters while encouraging the overly cautious that with appropriate safeguards, a start can be made. 